\title{
IMPROVING EMISSION-COMPUTED-TOMOGRAPHY QUANTIFICATION BY COMPTON-SCATTER REJECTION THROUGH OFFSET WINDOWS
}

\author{
Kenneth F. KORAL, Neal H. CLINTHORNE and W. Leslie ROGERS \\ Division of Nuclear Medicine, University of Michigan, Ann Arbor, Michigan 48109, USA
}

\begin{abstract}
Historically in nuclear medicine the acceptance window for total-energy pulse-height has been set to maximize contrast and resolution in the image. Recently, there has been increased emphasis on quantification of digital images to calculate, for example, left ventricular volume or tumor radiation absorbed dose. In this paper, we consider specifically what improvements can be made in emission computed tomography simply by employing a window which is offset to the high-energy side of the photopeak in order to reduce the contribution of Compton scattered gamma-rays. The window is offset so as to reduce the count rate for a source in air by $20 \%$. Two lucite phantoms were measured. One was a short cylinder filled with a uniformly-distributed solution of ${ }^{99 \mathrm{~m}} \mathrm{Tc}$ in water. The other was a head phantom with a $0.6 \mathrm{~cm}^{3}$ "tumor" containing ${ }^{99 \mathrm{~m}} \mathrm{Tc}$. Water surrounding the tumor could be nonradioactive or contain a dilute background activity. Absolute calibration was accomplished by imaging the simulated tumor in the air-filled phantom. With the offset window, calculated tumor activity is only $3 \%$ and $7 \%$ high without and with background respectively compared to $20 \%$ and $26 \%$ high with the symmetric window. However, total activity of the entire slice is still $37 \%$ high even without background. For the cylinder containing uniform activity, the error in specific activity drops from $30 \%$ to $18 \%$ with the offset window. Therefore, an asymmetric window significantly improves quantification, and for certain cases such as an isolated tumor, may be sufficiently accurate without further correction.
\end{abstract}

\section{Introduction}

Historically in nuclear medicine imaging of gammaray sources, the acceptance window for total energy pulse-height has been set to maximize contrast and resolution in the image [1]. Recently, there has been increased emphasis on quantification of digital images for applications such as determining the volume of the left ventricle by count-based methods [2] or calculating the radiation absorbed dose delivered to tumors after the administration of therapeutic amounts of a radioisotope [3-5].

Two related problems exist in seeking accurate quantification: How to correct for attenuation of gamma-rays as they leave the body and how to account for gammarays which have been Compton scattered in the body but which still fall within the energy acceptance window. In planar imaging, Siegel et al. [6] have recently advocated correcting the data with an attenuation factor which takes into account the acceptance of Compton scattered gamma-rays. Their attenuation factor is thus less than the linear attenuation coefficient, $u$, based on total cross section. For single-photon emission computed tomography (SPECT), three distinct approaches can be identified: One is to choose an appropriate value for $u$ empirically [7]; another is to use the good geometry $u$ but to subtract from the projection data $[8,9]$ or the reconstructed image [10] a fraction of the data or image obtained from a second window which is set in the Compton region of the pulse-height spectrum; and the third is to accomplish a reconstruction which explicitly takes into account the probability of Compton scattering [9].

In this study, we investigate the improvements in SPECT quantification that are made by rejecting a larger fraction of the Compton scattered gamma-rays through the use of a single window of set to the high energy side of the photopeak. We assume a $u$ based on total cross section of the uniformly attenuating medium in the experiments.

\section{Methods}

\subsection{Phantoms}

Two phantoms were employed. One was a cylinder filled with a uniformly distributed solution of ${ }^{99 \mathrm{~m}} \mathrm{Tc}$ in water. The other was a lucite head phantom with an entrance port for the insertion of a nearly spherical lucite "tumor" containing ${ }^{99 m} \mathrm{Tc}$ radioactive solution. This phantom was filled with water to simulate attenuation in the head which is generally held to be fairly uniform except for the skull. Measurements were made with and without a background activity added to the water.

\subsection{Imaging device}

The imaging device employed was the high-resolution single-slice SPRINT head tomograph [11]. Briefly, 
it is a tomograph with discrete sodium iodide detectors arranged in a fixed ring $70 \mathrm{~cm}$ in diameter. A complete set of fanbeam projections for the $20 \mathrm{~cm}$ field-of-view is acquired by means of a rotating lead aperture ring containing 8 slits. Slit width for the high resolution ring we employed here is $3.2 \mathrm{~mm}$. Converging cross-plane collimation is achieved with lead foil rings sandwiched between tapered styrofoam spacers.

The pulse-height windows have a fixed width of approximately $20 \%$. The pulse-height settings are determined using a point source in air as follows. First, the windows for all detectors are stepped from a maximum energy setting to a minimum energy setting under microprocessor control, and those settings which give the maximum count rate for each detector are stored as the symmetric windows. On a second pass, the windows are stepped upward starting at the symmetric window settings until the count rate drops to $80 \%$ of the maximum count rate for each detector.

\subsection{Calibration}

Absolute calibration was accomplished by imaging the simulated tumor in the air-filled phantom. The tumor had an internal volume of $0.6 \mathrm{~cm}^{3}$ and an ellipsoidal shape. The outer dimensions of two major axes were $12.7 \mathrm{~mm}$ and that of the minor axes was $9.0 \mathrm{~mm}$. Its activity was determined with a dose calibrator. Three contiguous planes were obtained by translating the source along the axis of slit rotation in $1 \mathrm{~cm}$ increments. Since the tumor was simulating a point source in air and the high window is defined and measured to be one that reduces the count rate of a point source in air by $20 \%$, the same results, reduced by $20 \%$, were used to calibrate the high-window measurements.

\subsection{Measurements}

The noncalibration measurements were of three forms and each was made with both the symmetric and the high window.

(1) The head phantom, with the radioactive brain tumor in place, was filled with water. The tumor was in an asymmetric position about $4 \mathrm{~cm}$ from the nearest edge at a level such that the head outline was an ellipse with major axis $18.65 \mathrm{~cm}$ and minor axis $14.9 \mathrm{~cm}$. It was imaged for contiguous planes $1 \mathrm{~cm}$ apart until source activity disappeared. It is to be noted that because of significant Compton scattering, five planes were required whereas only three were needed for the same source position in air.

(2) A uniform background source activity amounting to $0.26 \mu \mathrm{Ci} / \mathrm{cm}^{3}$ was added to the water in the head phantom to simulate activity outside the tumor and the above measurement repeated.

(3) The $19.05 \mathrm{~cm}$ diameter by $5.08 \mathrm{~cm}$ long cylin- drical phantom filled with water containing 1.18 $\mu \mathrm{Ci} / \mathrm{cm}^{3}$ was imaged in a single median plane.

In addition, a test was performed on the calibration source to see if finer sampling in the axis-of-rotation direction had an effect on the computed results. Planes were allowed to overlap by taking them at $0.5 \mathrm{~cm}$ separation instead of the usual $1.0 \mathrm{~cm}$ separation. The plane thickness was unchanged.

\subsection{Reconstruction algorithm}

All images were reconstructed by CSIM, a compensated simultaneous updating method [12]. The algorithm is an iterative least-squares-type algorithm in which the error correction is determined experimentally for a given class of problems and then fixed. This avoids the actual least squares calculation in the inner loop of the program. The forward projection along each ray is accomplished using a linked list of pixel weights. For each pixel on the diverging finite-width ray, these weights represent the product of detection solid angle and attenuation factor. They are thus equivalent to the probabilities for detection which are an important part of the Shepp and Vardi maximum-likelihood algorithm [13]. For the air measurement, the attenuation factor is taken as zero. For the water-filled phantoms, the attenuation factor is calculated from the distance between the pixel and the edge of the phantom multiplied by the total attenuation coefficient of $99 \mathrm{~m} \mathrm{Tc}$ in water, 0.15 $\mathrm{cm}^{-1}$.

Inspection of two measures of convergence indicated that 4 iterations of the above algorithm was sufficient. The first was the difference between the true projection data and the forward projection of the image. The second was the average change in the image pixel values. Although these measures were still changing after 4 iterations, the rate of change had decreased considerably from the initial rate of change and the fractional change over the last iteration was similar for the different objects.

\subsection{Analysis}

The method chosen for quantification involved summing the reconstructed strengths in specified regionsof-interest (ROIs).

For the calibration source and the tumor, the regionof-interest was determined by an automated second-derivative program. This program operated on an initial guess "seed" region drawn by hand once for a given object and both window settings. The parameters were set to choose the positive maximum after the zero crossing of the second derivative to demarcate the edge thus producing a region large enough to include the tail strength produced by finite resolution. Then the unknown tumor activity, $A_{\mathrm{t}}$, is found from the calibration 
point source activity $A_{\mathrm{p}}$, by

$$
\frac{A_{\mathrm{t}}}{A_{\mathrm{p}}}=\frac{S_{\mathrm{t}}}{S_{\mathrm{p}}} \text {, }
$$

where $S_{1}$ is the total strength of the tumor within a three-dimensional ROI (that is, the result of a double sum over planes and pixels) and $S_{\mathrm{p}}$ is the same for the calibration source. It is assumed that the imaging time for each plane and each case are the same and that tumor results with a given window are compared to calibration results with the same window.

For the uniform cylindrical phantom, the regionsof-interest over the entire source or over specified areas were drawn by hand. The relationship of the specific activity, $\mathscr{A}_{0}$, of the phantom to $A_{\mathrm{p}}$ is

$$
\frac{\mathscr{A}_{0}}{A_{\mathrm{p}}}=\frac{\mathscr{S}_{\mathrm{c}}}{d l^{2} S_{\mathrm{p}}},
$$

where $\mathscr{A}_{0}$ is the specific activity of the cylinder for the ROI considered, $\mathscr{S}_{\mathrm{c}}$ is the average cylinder strength per pixel in ROI considered for the median plane, $d$ is the plane thickness and $l^{2}$ is the area of a pixel.

\section{Results}

For the cylinder, the source-decay-corrected count rate with the high window was $70.6 \%$ of that with the symmetric window. For a total of 5 planes centered on the tumor, the same fraction was $67.5 \%$ for the head phantom with no background. The ratios are smaller than the $80 \%$ ratio for a point source in air because Compton scattering is now present and is being reduced by a greater factor.

Table 1 shows the results of tumor quantification both without and with uniform background. The true activities have been calculated by taking into account isotope decay. It is seen that with the symmetric window the value calculated is $20 \%$ and $26 \%$ too high without and with background respectively. The difference between the two values is reasonable as the background activity should increase Compton scattering. It is also seen in table 1 that changing to a high window leads to a value that correctly reproduces the tumor activity with no background present to within an estimated experimental error of $\pm 5 \%$. The interpretation is that, in this case, the offset window has reduced the number of Compton-scattered photons which lead to reconstructed activity within the tumor ROI to the extent that this activity is now on the order of the experimental uncertainty. With the given level of background, the error is $7 \%$ and thus slightly outside the estimated random error. The total background activity was more than twice the total tumor activity but, due to the much larger volume of the head, the background concentration in $\mu \mathrm{Ci} / \mathrm{cm}^{3}$ was only $0.05 \%$ that in the tumor. With higher background concentrations, the tumor quantification errors would be larger.

The Compton-scattered events have not been completely eliminated, of course, by the high window even in the case of no background. As shown in table 2, they are present so as to make the total measured activity over the complete cross section, summed over five planes, $175 \%$ of the true tumor value with the symmetric window and $137 \%$ with the high window.

Table 3 shows the results of quantifying the cylindrical phantom image using the point-source-in-air standard. The values are given as a function of regionof-interest with the window setting as a parameter. The middle ROI is a circle at the center enclosing $24 \%$ of the area. The edge ROI is the remaining $76 \%$ of the area. In all cases, the calculated specific activities are too high. Changing from a symmetric window to a high window reduces the error for all three regions-of-interest. For the total ROI, it is $30 \%$ too large for the symmetric window and $18 \%$ too large for the high window.

It is also seen that the error is larger in the middle of the circular region than at the edges. This result is consistent with the ${ }^{99 \mathrm{~m}} \mathrm{Tc}$ simulation results of Egbert and May [14] which showed that for a uniform-strength cylinder, Compton scattered photons lead to reconstructions with a high center and lower values at the edges.

Table 4 shows the quantification of the uniform background concentration and its true value both planeby-plane and as an average over planes. The calculated values of $\mathscr{A}_{0}$ were computed from eq. (2) with $\mathscr{P}_{\mathrm{c}}$ being the average background strength per pixel excluding the tumor ROI for the given plane. As in the case of

Table 1

\begin{tabular}{|c|c|c|c|c|}
\hline \multirow[t]{2}{*}{ Case } & \multirow[t]{2}{*}{ Window } & \multicolumn{2}{|c|}{ Activity $(\mu \mathrm{Ci})$} & \multirow{2}{*}{$\begin{array}{l}\text { Error } \\
(\%)\end{array}$} \\
\hline & & True & Calculated & \\
\hline \multirow[t]{2}{*}{ Water } & symmetric & 515 & 619 & +20 \\
\hline & high & 463 & 476 & +3 \\
\hline \multirow[t]{2}{*}{ Water with background ${ }^{\text {a) }}$} & symmetric & 372 & 470 & +26 \\
\hline & high & 402 & 430 & +7 \\
\hline
\end{tabular}

Tumor quantification

a) Total background activity distributed throughout head was twice the activity within the tumor. 
Table 2

Reconstructed activity for entire head cross section as function of plane and window. No water background activity

\begin{tabular}{lcc}
\hline $\begin{array}{l}\text { Plane location } \\
\text { relative to } \\
\text { tumor }(\mathrm{cm})\end{array}$ & Activity $(\mu \mathrm{Ci})$ & \\
\cline { 2 - 3 } & Symmetric window & High window \\
+2 & 39 & 24 \\
+1 & 100 & 65 \\
0 & 568 & 474 \\
-1 & 160 & 67 \\
-2 & 36 & 22 \\
Total & 903 & 652 \\
Total as \% of true & $+175 \%$ & $+137 \%$ \\
$\quad$ tumor activity & & \\
\hline
\end{tabular}

the circular cylinder, the calculated values are too high due to Compton scattering. The error averaged over planes is reduced from $81 \%$ to $45 \%$ by employing the high window. The listing by location of the plane relative to the tumor shows the error to be largest in the plane of the tumor. This result also is in agreement with the findings of Egbert and May that the reconstructed Compton scatter image totals the most in the plane of the source and decreases as the axial distance from true source to plane of interest increases [14,15].

Doubling the axial sampling increased the specific activity, $\mathscr{A}_{0}$, for the cylinder by only $1.2 \%$ of the given value. It was concluded that the axial sampling used in the measurements above was sufficient for our purposes.

\section{Discussion}

Present methods of correcting for attenuation and Compton scattering of gamma-rays in carrying out quantification are often accurate for a specific model or may require a difficult calculation for each patient. The buildup method of Siegel et al. [6] has only been shown to be accurate under the conditions (1) that the unknown is present in the same body phantom which was used for the measurements to specify the parameters of the method, and (2) that there is no background to simulate tissue uptake. The fraction of the Comptonscatter image which is to be subtracted from the primary image in the method of Jaszczak et al. varies between 0.66 and 0.47 as a line source in a uniform, $22 \mathrm{~cm}$ diameter cylinder changes its position by $8 \mathrm{~cm}$ [9].

And we foresee that, to accurately carry out a unified reconstruction as also proposed by Jaszczak, one would need to know the complete body outline, as well as the attenuation and scattering cross section and the background concentration at all interior points.

On the other hand, the asymmetric window method also has problems. It clearly approaches complete accuracy in the mean only as the count rate approaches zero.

Table 3

Cylindrical phantom quantification as function of region-of-interest and window setting

\begin{tabular}{|c|c|c|c|c|c|c|}
\hline \multirow[t]{3}{*}{ ROI } & \multicolumn{6}{|l|}{ Specific activity } \\
\hline & \multicolumn{3}{|l|}{ Symmetric window } & \multicolumn{3}{|l|}{ High window } \\
\hline & True $\left(\mu \mathrm{Ci} / \mathrm{cm}^{3}\right)$ & Calculated $\left(\mu \mathrm{Ci} / \mathrm{cm}^{3}\right)$ & Error $(\%)$ & True $\left(\mu \mathrm{Ci} / \mathrm{cm}^{3}\right)$ & Calculated $\left(\mu \mathrm{Ci} / \mathrm{cm}^{3}\right)$ & Error $(\%)$ \\
\hline Middle & 1.18 & 2.00 & +69 & 1.15 & 1.72 & +49 \\
\hline Total & 1.18 & 1.54 & +30 & 1.15 & 1.36 & +18 \\
\hline Edge & 1.18 & 1.40 & +18 & 1.15 & 1.25 & +8 \\
\hline
\end{tabular}

Table 4

Uniform background quantification as function of plane and window setting

\begin{tabular}{|c|c|c|c|c|c|c|}
\hline \multirow{3}{*}{$\begin{array}{l}\text { Plane location } \\
\text { relative to } \\
\text { tumor }(\mathrm{cm})\end{array}$} & \multicolumn{6}{|l|}{ Specific activity } \\
\hline & \multicolumn{3}{|c|}{ Symmetric window } & \multicolumn{3}{|l|}{ High window } \\
\hline & True $\left(\mu \mathrm{Ci} / \mathrm{cm}^{3}\right)$ & Calculated $\left(\mu \mathrm{Ci} / \mathrm{cm}^{3}\right)$ & Error (\%) & True $\left(\mu \mathrm{Ci} / \mathrm{cm}^{3}\right)$ & Calculated $\left(\mu \mathrm{Ci} / \mathrm{cm}^{3}\right)$ & Error $(\%)$ \\
\hline+2 & 0.245 & 0.38 & +56 & 0.265 & 0.32 & +20 \\
\hline+1 & 0.245 & 0.45 & +82 & 0.265 & 0.37 & +39 \\
\hline 0 & 0.245 & 0.53 & +114 & 0.265 & 0.54 & +104 \\
\hline-1 & 0.245 & 0.45 & +84 & 0.265 & 0.36 & +37 \\
\hline-2 & 0.245 & 0.41 & +68 & 0.265 & 0.33 & +25 \\
\hline Average & 0.245 & 0.44 & +81 & 0.265 & 0.38 & +45 \\
\hline
\end{tabular}


However, we have shown here that at least with a low background concentration, the quantification of a small tumor can be accurate to better than a $10 \%$ error. Therefore, it appears that the method of choice will depend on the situation and that the simple offset windows method may be useful for calculating dosimetry of tumors with high target to nontarget uptake.

\section{Acknowledgment}

This work was supported by NIH grant Nos. 1 ROI GM28305-01 and 5T32-CA09015-05. The authors thank Diane Vecellio for secretarial assistance in preparation of the manuscript.

\section{References}

[1] R.N. Beck, L.T. Zimmer, D.B. Charleston, P.B. Hoffer and $N$. Lembares, in: Semiconductors in the Future of Nuclear Medicine (Society of Nuclear Medicine, 1971) pp. 92-113.

[2] M.A. Rabinovitch et al. Radiology 150 (1984) 813.

[3] H.R. Maxon, S.R. Thomas, V.S. Hertzberg et al., N. Engl. J. Med. 309 (1983) 937
[4] P.K. Leichner, J.L. Klein, S.S. Siegelman, D.S. Ettinger and S.E. Order, Cancer Treatment Reports 67 (1983) 647.

[5] R.S. Adler, K.F. Koral, J.E. Carey, R.C. Kline and W.H Beierwaltes, Med. Phys. 9 (1982) 497.

[6] J.A. Siegel, R.K. Wu and A.H. Maurer, J. Nucl. Med. 26 (1985) 490.

[7] D. Osborne, R. Jaszczak, R.E. Coleman, K. Greer and M. Lischko, J. Nucl. Med. 23 (1982) 446.

[8] R.J. Jaszczak, K.L. Greer, C.E. Floyd Jr, C.C. Harris and R.E. Coleman. J. Nucl. Med. 25 (1984) 893.

[9] R.J. Jaszczak. C.E. Floyd Jr and R.E. Coleman, IEEE Trans. Nucl. Sci. NS-32 (1985) 786.

[10] J.M. Sanders and N.M. Spyrou, Nucl. Instr. and Meth. 221 (1984) 93

[11] W.L. Rogers, N.H. Clinthorne, J. Stamos, K.F. Koral, R. Mayans, G.F. Knoll, J. Juni. J.W. Keyes Jr and B.A Harkness, J. Nucl. Med. 25 (1984) 1013.

[12] W.P. Snapp. PhD Thesis, University of Michigan, Ann Arbor (1979).

[13] L.A. Shepp and Y. Vardi, IEEE Trans. Med. Imaging MI-1 (1982) 113.

[14] S.D. Egbert, PhD Thesis, University of Illinois, UrbanaChampaign (1980).

[15] S.D. Egbert and R.S. May, IEEE Trans. Nucl. Sci. NS-27 (1980) 543. 